\title{
IMPROVING THE ORDINAL INCONSISTENCY OF PAIRWISE COMPARISON MATRICES
}

\author{
Ludmil Mikhailov* \\ Manchester Business School \\ Univers ity of Manchester, UK \\ E-mail: $\underline{\text { ludi.mikhailov@,manchester.ac.uk }}$ \\ Sajid Siraj \\ School of Computer Science \\ Univers ity of Manchester, UK \\ E-mail: $\underline{\text { sajidsiraj@gmail.com }}$
}

\begin{abstract}
This paper investigates the effects of non-transitive judgments on the consistency of pairwise comparison matrices and proposes a heuristic algorithm for identifying and eliminating the ir ordinal inconsistencies. The algorithm is based on graphical representation of the comparison matrices and identifies the edges in the digraph, which are mostly responsible for three-way cycles, representing the ordinal incons istencies. The algorithm tries to minimize the number of edge reversals and provides results, similar to those obtained by an optimization method.
\end{abstract}

Keywords: Pairwise Comparisons, Inconsistency, Non-transitive Judgements, Decision Analysis, Heuristics

\section{Introduction}

Pairwise comparisons are a vital part of the prioritisation procedure in the Analytic Hierarchy Process (AHP), which provides a comprehensive and rational framework for structuring a decision problem (Saaty, 1980). In the AHP, the pairwise judgements are structured in pairwise comparison matrices (PCM), and some prioritisation procedures are applied to derive a corresponding priority vector. If the comparison judgements are cardinally consistent, then the PCM are also consistent, and all prioritisation methods give the same result. However, in the case of ordinal or cardinal inconsistent judgements, different prioritisation methods derive different priority vectors.

The AHP does not require transitivity of the comparison judgements. Saaty's CR index measures the cardinal inconsistency of the judgements, but does not capture the ordinal inconsistency. Ordinal inconsistency always implies cardinal inconsistency, however, the converse does not hold. Generally, if the comparison judgements and the corresponding PCM are ordinally inconsistent, the level of their cardinal inconsistency is considerably high; therefore the AHP implicitly presumes that satisfying the CR test may significantly reduce the chances of ordinal inconsistency. However, there are examples in the literature where matrices that satisfy the CR criterion can also be ordinarily inconsistent (Jensen and Hicks, 1993; Kwiesielewicz and van Uden, 2004).

The removal of intransitivities can be formulated as a non-linear integer programming problem problem (Mikhailov et al, 2010). However, its solution requires applying a complex optimisation procedure and is

\footnotetext{
* Corresponding author
} 
rather difficult from computational point of view. This paper proposes a heuristic procedure for improving the overall consistency of PCM by detecting and modifying inconsistent ordinal judgements. The heuristic algorithm achieves almost identical results to the optimisation algorithm; however, it does not require applying numerical methods and is much more efficient from a computational viewpoint.

\section{Pairwise Comparison Matrices}

Consider a prioritization of $n$ elements $E_{1}, E_{2}, \ldots, E_{n}$ at the same level hierarchy. The decision maker (DM) assesses the relative importance of any two elements $E_{i}$ and $E_{j}$ by providing a comparison judgment $a_{i j}$, specifying by how much $E_{i}$ is preferred/not preferred to $E_{j}$. The AHP method structures any set of comparison judgements in a positive reciprocal PCM, $A=\left[a_{i j}\right]$. Then a priority vector $w=\left(w_{1}, w_{2}, \ldots, w_{n}\right)^{T}$ could be obtained from $A$, by applying some prioritization method (Choo and Wedley, 2004).

\subsection{Consistency of PCM}

The judgments of DMs are cardinally consistent if $a_{i j}=a_{i k} * a_{k j}$ for all $i$ and $j$; where $j>k>i$ (Saaty 1980). When the judgments of the DM are perfectly consistent, then the judgements $a_{i j}$ have perfect values $a_{i j}=w_{i} / w_{j}$. In such a case, the PCM is said to be (perfectly) consistent. If the DM's judgements are cardinally inconsistent (i.e. $a_{i j} \neq a_{i k} * a_{k j}$ for some $i, j, k$ ) then the corresponding comparison matrix $A$ is said to be inconsistent and we have $a_{i j} \approx w_{i} / w_{j}$.

Saaty (1980) proposed a measure of consistency based on the properties of positive reciprocal matrices and defines a measure of consistency, called a Consistency Ratio $(C R)$. If the value of $C R$ is smaller than or equal to 0.1 , the estimated priority vector $w$ can adequately approximate the unknown preference vector $r$, therefore, the PCM is of acceptable inconsistency. However, if $C R>0.1$, the estimated priorities could be erroneous and the DMs should be asked to improve the consistency by revising the ir subjective judgments.

The ordinal consistency, which is also known as a transitivity condition between 3 elements, states that if $E_{i}$ is preferred to $E_{j}$ and $E_{j}$ is preferred to $E_{k}$, then $E_{i}$ should be preferred to $E_{k}$. Using the preference symbol $\rightarrow$, the ordinal consistency is represented as: if $E_{i} \rightarrow E_{j} \rightarrow E_{k}$ then $E_{i} \rightarrow E_{k}$. If however, $E_{k} \rightarrow E_{i}$ when $E_{i} \rightarrow E_{j} \rightarrow E_{k}$, then the preference judgements are ordinally inconsistent, or intransitive. Therefore, the ordinal incons istency could be defined as $E_{i} \rightarrow E_{j} \rightarrow E_{k} \rightarrow E_{i}$, which represents a circular triad of preferences (Kendall and Smith 1940). In terms of PCM, the preference relation $E_{i} \rightarrow E_{j}$ means that $a_{i j}>1$. Therefore, the ordinal inconsistency $E_{i} \rightarrow E_{j} \rightarrow E_{k} \rightarrow E_{i}$ means that the corresponding judgements are $a_{i j}>1, a_{j k}>1, a_{k i}>1$.

\subsection{Graphical representation of PCM}

The relationships between the elements of a PCM in the case of preference dominance can be depicted by a directed graph (digraph), where the intransitive relationship $E_{i} \rightarrow E_{j} \rightarrow E_{k} \rightarrow E_{i}$ is represented as a three-way cycle between the three elements (Kendall, 1955). 
Consider a problem with 5 comparison elements, where the DM provides the following PCM:

$$
\begin{aligned}
& \begin{array}{lllll}
E_{1} & E_{2} & E_{3} & E_{4} & E_{5}
\end{array} \\
& A=\left[\begin{array}{ccccc|l}
1 & 7 / 4 & 3 / 4 & 5 / 2 & 7 / 4 \\
4 / 7 & 1 & 3 / 4 & 9 / 4 & 9 / 4 \\
4 / 3 & 4 / 3 & 1 & 3 / 4 & 3 / 4 \\
2 / 5 & 4 / 9 & 4 / 3 & 1 & 5 / 8 \\
4 / 7 & 4 / 9 & 4 / 3 & 8 / 5 & 1
\end{array}\right] \begin{array}{l}
E_{1} \\
E_{2} \\
E_{4} \\
E_{5}
\end{array}
\end{aligned}
$$

In the digraph of this matrix, shown in Fig. 1, each comparis on element $E_{i}$ is represented as a node and the judgements as edges between the nodes. For example, the directed edge from $E_{1}$ to $E_{2}$ shows that $E_{1}$ is preferred to $E_{2}$, whereas the weight of the edge represents the intensity of the preference, which is equal to the value of the pairwise comparison judgement between these two elements, $a_{12}=2$. It can be seen that the diagraph contains four three-way cycles: $E_{1} \rightarrow E_{4} \rightarrow E_{3} \rightarrow E_{1}, \quad E_{1} \rightarrow E_{5} \rightarrow E_{3} \rightarrow E_{1}$, $E_{2} \rightarrow E_{4} \rightarrow E_{3} \rightarrow E_{2}$ and $E_{2} \rightarrow E_{5} \rightarrow E_{3} \rightarrow E_{2}$. As the comparison judgements are intransitive and there are cycles in its digraph, the comparison matrix (1) is ordinally incons istent.

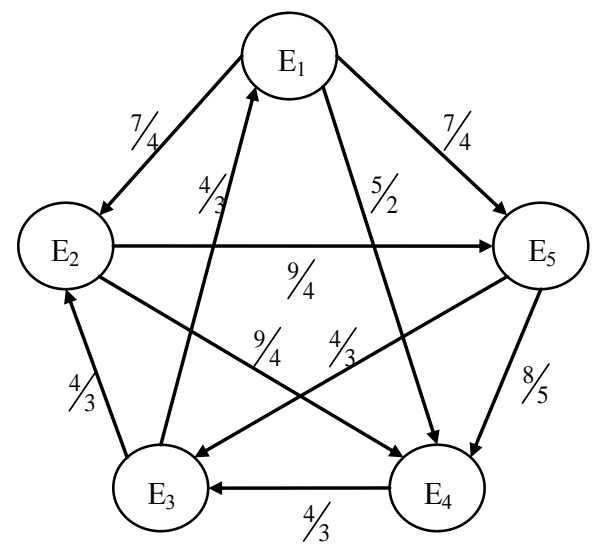

Fig. 1. Preference graph with cyclic judgments

In PCMs of dimension $n>3$, cycles with more than three ways may exist. For example, in the graph shown in Fig. 1, there is a four-way cycle $E_{1} \rightarrow E_{5} \rightarrow E_{4} \rightarrow E_{3} \rightarrow E_{1}$. However, it is important to note that if there are no three-way cycles in the digraph, then there are no cycles of higher order (Gass, 1998).

\subsection{Priority violations}

When $E_{i} \rightarrow E_{j}$, it is assumed that the priorities of the elements should preserve the preference direction, i.e. $w_{i}>w_{j}$. However, if $E_{j}$ receives a larger priority weight, $w_{j}>w_{i}$, then a priority violation occurs (Ali et al. 1986).

The number of violations $(N V)$ is defined as follows:

$$
N V(w)=\sum_{i=1}^{n-1} \sum_{j=i+1}^{n} v_{i j},
$$




$$
v_{i j}=\left\{\begin{array}{cc}
1, & \text { if } w_{i}>w_{j} \text { and } a_{i j}<1, \text { or } w_{i}<w_{j} \text { and } a_{i j}>1, \\
1 / 2, & \text { if } w_{i}=w_{j} \text { and } a_{i j} \neq 1, \text { or } w_{i} \neq w_{j} \text { and } a_{i j}=1, \\
0, & \text { otherwise. }
\end{array}\right.
$$

If the PCM is ordinally inconsistent, it is not possible to find a priority vector that satisfies all preference directions expressed by the ordinal comparisons and, therefore, there will always be priority violations.

The priority vectors $w$ for the PCM of this example, obtained by the Eigenvector method (EV), the Direct Least Squares (DLS), the Logarithmic Least Squares (LLS), the Logarithmic Least Absolute Value (LLAV) and the Logarithmic Absolute Error (LAE) (Choo and Wedley, 2004) are shown in Table 1.

Table 1. Priorities derived by different prioritisation methods

\begin{tabular}{lcccccc}
\hline Method & w1 & w2 & w3 & w4 & w5 & NV \\
\hline EV & 0.269 & 0.226 & 0.203 & 0.134 & 0.169 & 4 \\
DLS & 0.282 & 0.239 & 0.191 & 0.124 & 0.164 & 4 \\
LLS & 0.275 & 0.227 & 0.194 & 0.132 & 0.172 & 4 \\
LLAV & 0.303 & 0.253 & 0.139 & 0.120 & 0.186 & 3 \\
LAE & 0.283 & 0.255 & 0.187 & 0.113 & 0.162 & 4 \\
MNV & 0.350 & 0.286 & 0.086 & 0.136 & 0.143 & 2 \\
\hline
\end{tabular}

From the results it can be seen that the EV, the DLS and LLS methods derive priority vectors with the same ranking order $w_{1}>w_{2}>w_{3}>w_{5}>w_{4}$ (but with different intensities). This ranking has four violations, i.e. $w_{1}>w_{3}, w_{2}>w_{3}, w_{3}>w_{4}$ and $w_{3}>w_{5}\left(\right.$ as $\left.a_{13}<1, a_{23}<1, a_{34}<1, a_{35}<1\right)$. The LAE method generates a priority vector with a different rank order, which, however, also has four violations, whereas the LLAV method produces a ranking with only three violations. The priority vector with Minimum $N V(\mathrm{MNV})$ is obtained by applying an optimization procedure for minimizing (2). No prioritization method can obtain a vector with less than 2 priority violations.

It should be noted that the PCM (1) used in this example is of acceptable inconsistency, as its $C R=0.083$. Hence, by applying the $C R$ test only, the PCM will be classified as acceptable and the DM will not be asked to reconsider their judgements, therefore, regardless of the used prioritisation method, erroneous results will be obtained. It is obvious that the ordinal inconsistency is the main cause for obtaining priorities, which do not correspond to the DM's preferences. Therefore, the improvement of ordinal inconsistency by elimination of intransitivities could be regarded as the most important way to increase the accuracy of the decision-making process

\section{Heuristic Approach for Rectification of Intransitive Judgments}

Elimination of intransitive judgements can be formulated as an optimization problem to remove cycles by changing a minimal number of elements in the PCM (Mikhailov, 2010). However, in the case of pairwise comparison judgments, the available cardinal information can be additionally used to further sift the possible edges to be reversed.

Consider a partial preference digraph between any two elements $E_{i}$ and $E_{j}$. Let $\alpha$ be the number of times that $E_{i}$ is preferred over other elements in the overall digraph. Similarly, let $\beta$ be the number of times when $E_{j}$ is preferred over other elements. Fig. 2 shows a digraph, where $E_{i}$ is preferred to $E_{j}$ as 
the edge $v_{i j}$ is directed towards $E_{j}$. The element $E_{i}$ is also preferred to four other elements, therefore, its number of outflows is $\alpha=5$. Similarly, $E_{j}$ is preferred to three other elements, so it has three outflows.

Kendall and Smith (1940) showed that when $\alpha>\beta$ and the edge $v_{i j}$ is reversed, the number of threeway cycles in the overall preference digraph is increased. Moreover, when $\alpha<\beta$, reversing the direction of the edge $v_{i j}$ reduces the number of three-way cycles. This observation can be used to develop an iterative Heuristic algorithm, which can reduce both the intransitive elements and the number of threeway cycles.

At each iteration of the proposed Heuristic algorithm, the difference between the outflows of any two elements $E_{i}$ and $E_{j}$ is calculated and the edge, satisfying the condition $\max _{i, j}\left(\beta_{j}-\alpha_{i}\right)$ is reversed. In the case of multiple edges meeting this condition, the most inconsistent edge is to be reversed, using the cardinal consistency criterion $a_{i j}=a_{i k} a_{k j}$.

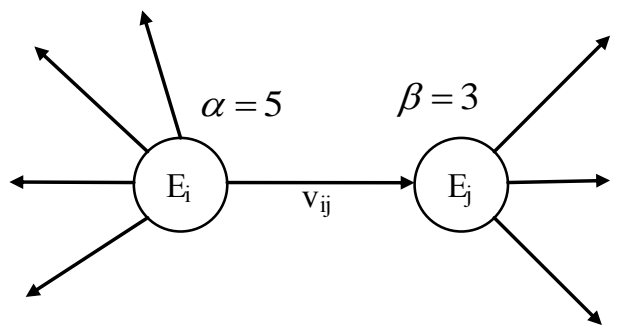

Fig. 2. Outflows for source and destination elements

Let $\gamma_{i j}$ be a value measuring the level of inconsistency contributed by $v_{i j}$ to the overall inconsistency of the pair-wise comparisons. The value of $\gamma_{i j}$ is calculated as the mean logarithmic deviation for all indirect judgments $a_{i k} a_{k j}$ from the direct judgement $a_{i j}$, i.e.

$$
\gamma_{i j}=\frac{1}{(n-2)} \sum_{k=1}^{n}\left(\log \left(a_{i j}\right)-\log \left(a_{i k} a_{k j}\right)\right), \text { where } k \neq i \neq j .
$$

Consider again the digraph of the 5-dimensional PCM, shown on Fig. 1. The values of $\alpha$ and $\beta$ for all its edges are listed in Table 2.

Table 2. The values of $\alpha, \beta$ and $\gamma$ for all edges of the digraph in Fig. 1 at the first iteration

\begin{tabular}{|c|c|c|c|c|}
\hline $\mathrm{V}$ & $\alpha$ & $\bar{\beta}$ & $\beta-\alpha$ & $\gamma$ \\
\hline$v_{12}$ & 3 & 2 & -1 & 0.61 \\
\hline $\mathrm{v}_{14}$ & 3 & 1 & -2 & 0.69 \\
\hline $\mathrm{v}_{15}$ & 3 & 2 & -1 & 0.69 \\
\hline $\mathrm{v}_{24}$ & 2 & 1 & -1 & 0.77 \\
\hline $\mathrm{v}_{25}$ & 2 & 2 & 0 & 0.89 \\
\hline $\mathrm{v}_{31}$ & 2 & 3 & 1 & 1.06 \\
\hline$v_{32}$ & 2 & 2 & 0 & 1.11 \\
\hline $\mathrm{v}_{43}$ & 1 & 2 & 1 & 1.12 \\
\hline $\mathrm{v}_{53}$ & 2 & 2 & 0 & 1.00 \\
\hline $\mathrm{v}_{54}$ & 2 & 1 & -1 & 0.35 \\
\hline
\end{tabular}


According to the algorithm, the edges with the maximum value of $\beta-\alpha$ should be identified. In this case, $v_{31}$ and $v_{43}$ are the edges with maximum value of $\beta-\alpha=1$. Therefore, the values of $\gamma_{31}$ and $\gamma_{43}$, shown in Table 2 are used to select the most inconsistent edge. As the level of inconsistency $\gamma_{43}$ is greater than $\gamma_{31}$, the edge $v_{43}$ should be reversed. In terms of the elements of the initial comparison matrix (1), we interchange the values of the corresponding comparison elements $a_{43}$ and $a_{34}$, so the updated values are $a_{43}=3 / 4$ and $a_{34}=4 / 3$, respectively. After the first iteration, two of the four cycles are removed from the original PCM. The two cycles remaining in the updated PCM are $E_{1} \rightarrow E_{5} \rightarrow E_{3} \rightarrow E_{1}$ and $E_{2} \rightarrow E_{5} \rightarrow E_{3} \rightarrow E_{2}$.

In the next iteration, the edges with the maximum value of $\beta-\alpha$ are identified for the updated PCM. The edge $v_{53}$ has the maximum value of $\beta-\alpha$ equal to 1 . The value of $\gamma$ is irrelevant in this iteration, as there are no other edges with such $\beta-\alpha$. Therefore, the comparison elements $a_{53}$ and $a_{35}$ are swapped and their new values become $a_{53}=3 / 4$ and $a_{35}=4 / 3$, respectively. Thus, the updated PCM has no three-way cycles and becomes transitive. Its $C R$ is improved to 0.055 from the original value of 0.083 . It can be shown that the obtained heuristic solution is equivalent to optimal solution to this problem, however, it is both much faster and simpler from a computation point of view.

\section{Conclusions}

This paper justifies the need to improve the ordinal consistency and proposes an efficient heuristic algorithm to eliminate the intransitivity of PCMs. The proposed approach does not replace the CR consistency test. It could be considered as an additional procedure for improving the overall consistency of the comparison judgements, and consequently, the quality of the decision making process.

\section{REFERENCES}

Saaty, T. (1980). The Analytic Hierarchy Process, Planning, Piority Setting, Resource Allocation, New York: McGraw-Hill.

Ali, I., Cook, W., \& Kress, M. (1986). On the Minimum Violations Ranking of a Tournament. Management Science, 32(6), 660-672.

Choo, E., \& Wedley, W. (2004). A Common Framework for Deriving Preference Values from Pairwise Comparison Matrices. Comput. Oper. Res., 31(6), 893-908.

Jensen, R., \& Hicks, T. (1993). Ordinal data AHP analysis: A proposed coefficient of consistency and a nonparametric test. Mathematical and Computer Modelling, 17(4-5), 135-150.

Gass, S. (1998). Tournaments, Transitivity and Pairwise Comparison Matrices. The Journal of the Operational Research Society, 49(6), 616-624.

Kendall, M. (1955). Further Contributions to the Theory of Paired Comparisons. Biometrics, 11(1), 4362.

Kendall, M., \& Smith, B. (1940). On the Method of Paired Comparisons. Biometrika, 31(3/4), 324-345.

Kwiesielewicz, M., \& van Uden, E. (2004). Inconsistent and contradictory judgements in pairwise comparison method in the AHP. Comput. Oper. Res., 31(5), 713-719. 
Mikhailov, L., Siraj, S., \& Keane, J. (2010). Ordinal Inconsistency of Pairwise Comparison Judgements, EDSI'2010, Barcelona, Spa in. 